\title{
RECALCITRANT HYPOCALCEMIA IN A LACTATING WOMAN AFTER TOTAL THYROIDECTOMY FOR PAPILLARY THYROID CARCINOMA
}

\author{
Amy Anne D. Lassig, MD, ${ }^{1}$ Paul E. Donatelli, MD, ${ }^{2}$ Theodoros N. Teknos, MD ${ }^{1}$ \\ ${ }^{1}$ Department of Otolaryngology-Head and Neck Surgery, University of Michigan, Ann Arbor, MI. \\ E-mail: donat037@umn.edu \\ ${ }^{2}$ Department of Internal Medicine, Roper Hospital, Charleston, SC
}

Accepted 13 November 2009

Published online 8 March 2010 in Wiley Online Library (wileyonlinelibrary.com). DOI: 10.1002/hed.21344

\begin{abstract}
Background. The risk of hypocalcemia after total thyroidectomy is well-described in the literature. Recalcitrant hypocalcemia after thyroidectomy in a lactating woman is uncommon and may require multiple strategies to correct.

Methods. We present a case report of a lactating woman with papillary thyroid carcinoma requiring total thyroidectomy and neck dissections.

Results. Postoperatively the patient suffered from recalcitrant hypocalcemia which necessitated multiple medical maneuvers to rectify. The medical treatment required to correct the calcium levels is described herein.

Conclusion. With the incidence of thyroid cancer on the rise and the increased identification of thyroid lesions during pregnancy, the number of lactating women undergoing thyroidectomy will likely increase. Surgeons performing thyroid surgery in this patient population should be aware of the risk of severe hypocalcemia and the tools necessary to correct it. () 2010 Wiley Periodicals, Inc. Head Neck 33: 920-922, 2011
\end{abstract}

Keywords: thyroidectomy; papillary thyroid carcinoma; hypocalcemia; lactation; thyroid cancer

Well-differentiated thyroid cancer is a well-described head and neck malignancy. The incidence is on the rise in the United States, and as such, thyroidectomy is increasingly required to treat this disease. ${ }^{1}$ The complications of thyroidectomy are documented in the literature. They include clinically significant events of hypoparathyroidism and recurrent laryngeal nerve injury. Hypocalcemia secondary to hypoparathyroidism can be transient or long-lasting, and not infrequently requires treatment in the postoperative period. This complication, however, is generally shortlived and not difficult to correct. The affects of lactation on calcium metabolism are likewise described in the literature. These 2 processes have been little described in concert, however, to result in recalcitrant hypocalcemia. Thus, we present a case of a young lactating woman who underwent total thyroidectomy and

Correspondence to: A. A. D. Lassig

(C) 2010 Wiley Periodicals, Inc. neck dissections with resulting recalcitrant hypocalcemia.

\section{CASE REPORT}

A 24-year-old otherwise healthy woman presented to an outside physician with a right neck mass noted during her most recent pregnancy. The mass was enlarging, and a fine-needle aspiration (FNA) was positive for papillary thyroid carcinoma (PTC). She subsequently underwent imaging with a CT scan of the neck with contrast, ordered by her outside primary care physician who was unfamiliar with PTC and the contraindication to iodinated dye. The scan revealed bilateral lymphadenopathy, massive on the right, with a small nodule in the superior pole of the right hemithyroid. There was no evidence of distant disease. The patient was then referred to our practice and scheduled for surgery. She was found to be healthy, with the exception of iron deficiency anemia. The patient was 2 months postpartum on her referral to our clinic and had delivered and breastfed 3 children over the previous 4 years.

The patient underwent a total thyroidectomy with recurrent laryngeal nerve monitoring, right modified radical neck dissection (sparing cranial nerve XI and the sternocleidomastoid muscle), left selective neck dissection levels 1 through 4, bilateral paratracheal node dissection, and superior mediastinal dissection. Intraoperative findings included massive lymphadenopathy in right levels 1 through 5 and gross lymphadenopathy in all other levels dissected. The thyroid itself was relatively normal with a small firm mass in the right superior pole. On each side, 1 parathyroid gland was visualized and preserved; however, such identification was difficult due to the significant confluence of small lymph nodes surrounding the gland. Pathology revealed papillary thyroid carcinoma in the right lobe $(1.2 \mathrm{~cm})$ with diffuse intrathyroidal involvement and capsular invasion. Positive nodes were appreciated in all nodal regions. 
The patient was admitted postoperatively for routine care and monitoring. Ionized calcium obtained in the recovery room was normal at $1.15 \mathrm{mmol} / \mathrm{L}$ (ref. 1.12-1.30); however, 8 hours later it decreased to 1.05. She was monitored every 8 hours, and her ionized calcium continued to decline to be $1.02,0.92$, and 0.88 over the next 24 hours. The patient was symptomatic from these levels with perioral and distal extremity paresthesias. She was started on oral calcium with IV supplementation.

Over the next 5 postoperative days (POD), the patient's ionized calcium continued to be monitored every 8 hours. She continued to have significant symptomatic hypocalcemia despite aggressive medical management. The patient was also noted to have mild hypomagnesemia and hyperphosphotemia. Her calcium levels ranged from 0.83 to 1.27 (outlier), with a mean of 0.94 and a median of 0.92 . During this time period, aggressive medical management was required to correct the patient's calcium levels, as depicted in Table 1.

Highlights of the medical management to correct the patient's hypocalcemia are described as follows. In brief, treatment began with oral calcium supplementation in the form of calcium carbonate. Calcium and magnesium supplementation were given IV on PODs 1-5 when levels were deemed dangerously low from a cardiac standpoint. Vitamin D (1,25-dihydroxycholecalciferol) was added on POD 2 to increase intestinal and renal absorption, and the dose was increased on POD 3 and POD 5. Likewise oral calcium carbonate was increased on POD 2 and POD 3 and then changed to calcium citrate on POD 4. Calcium citrate has been found to have greater bioavailability after oral ingestion than calcium carbonate. ${ }^{2,3}$ The dose of calcium citrate was increased on POD 5. Endocrinology was consulted on POD 4. Oral magne- sium was started on POD 4 and increased on POD 5. The patient had continued active lactation to this point in her hospital stay, and on POD 6 this was stopped. Bromocriptine was also added to suppress lactation. On POD 7 and POD 8, her ionized calcium levels began consistently trending up (0.86 up to 1.08). On POD 8, T3 was added to her regimen. On POD 8, hydrochlorothiazide was added to reduce calciuria. By POD 9, the patient's ionized calcium levels were near normal (1.06-1.11), and she was asymptomatic. She was thus discharged to home on calcium citrate, calcitriol, bromocriptine, T3, magnesium, and hydrochlorothiazide. Discharge plans included weekly calcium monitoring and close endocrine follow-up. At her 8-month follow-up, the patient continued to require similar levels of calcium, calcitriol, and magnesium replacement with the addition of levothyroxine.

\section{DISCUSSION}

Hypocalcemia after total thyroidectomy is welldescribed in the literature. It is thought to be the most common complication after this surgery, occurring in up to $30 \%$ of patients, but transiently in all but $3 \% .^{4}$ Severely recalcitrant hypocalcemia after total thyroidectomy is exceedingly rare, and its association with the lactating woman has been little described in the literature. Mineral metabolism in the pregnant and lactating woman is remarkably complex to allow for adequate nutrition of the fetus and newborn. Lactating women loose approximately 280 to $400 \mathrm{mg}$ of calcium daily through breast milk. Calcium demands during pregnancy are met through increased intestinal absorption mediated by 1,25dihydroxyvitamin D. Conversely, the large calcium demands of lactation are met via demineralization of

Table 1. Medical treatment for hypocalcemia by postoperative day.

\begin{tabular}{|c|c|c|}
\hline Postoperative day & Treatment changes & Dose \\
\hline 1 & Calcium carbonate & 1500 mg PO TID \\
\hline 2 & $\begin{array}{l}\text { Calcium carbonate } \\
1 \text { 25-dihydroxycholecalciferol (calcitriol) }\end{array}$ & $2 \mathrm{~g}$ PO TID \\
\hline 3 & $\begin{array}{l}\text { Calcium carbonate } \\
\text { 1,25-dihydroxycholecalciferol }\end{array}$ & $\begin{array}{l}2.5 \mathrm{~g} \text { PO TID } \\
0.5 \text { micrograms PO BID }\end{array}$ \\
\hline \multirow[t]{3}{*}{4} & Calcium carbonate discontinued & \\
\hline & Calcium citrate & $2.4 \mathrm{~g} P O \mathrm{QID}$ \\
\hline & Magnesium oxide & 800 mg PO TID \\
\hline \multirow[t]{3}{*}{5} & Calcium citrate & $2.85 \mathrm{~g}$ PO QID \\
\hline & 1,25-dihydroxycholecalciferol & 0.5 micrograms $\mathrm{PO}$ TID \\
\hline & Magnesium oxide & 1600 mg PO TID \\
\hline \multirow[t]{2}{*}{6} & Lactation stopped & \\
\hline & Bromocriptine & 1.25 mg PO TID \\
\hline 7 & No change & \\
\hline \multirow[t]{2}{*}{8} & T3 (liothyronine) & 12.5 micrograms $\mathrm{PO} Q \mathrm{QD}$ \\
\hline & Hydrochlorothiazide & $12.5 \mathrm{mg} P O \mathrm{QD}$ \\
\hline
\end{tabular}

Abbreviations: $P O$, prescribed orally; TID, taken $3 \times$ daily; $Q D, 1$ daily; $B I D, 2 \times$ daily; $Q I D, 4 \times$ daily.

${ }^{*} P O D$ 1-5: IV Calcium gluconate (4-7 g total daily dose) and IV magnesium (0-7 $\mathrm{g}$ total daily dose). 
the skeleton. This is mediated via parathyroid hormone-related protein (PTHrP) and via a low estrogen state. PTHrP is released from lactating mammary tissue to result in resorption of maternal skeleton calcium, increased renal tubular absorption of calcium, and secondary suppression of parathyroid hormone (PTH). ${ }^{5}$ This results in a "hungry bone syndrome" type picture in the lactating woman, which is thought to completely resolve after weaning. The complicated mechanisms of calcium homeostasis in the pregnant and lactating woman continue to be elucidated.

The above described patient presented to us having been pregnant 3 consecutive times over 4 years with continued lactation throughout. She thus presented in a long-term state of severely altered calcium metabolism, with continuing massive calcium demands by ongoing lactation. As such, the patient suffered from recalcitrant hypocalcemia when 2 of her parathyroid glands were inadvertently removed and the other 2 glands were devascularized. Multiple medical maneuvers were required to correct this. At the most basic level, calcium and vitamin D were added to replenish calcium levels and allow for intestinal and renal absorption of calcium. Magnesium was added because low levels can result in deranged synthesis and release of PTH and impaired systemic function of PTH. The patient stopped breast feeding, and bromocriptine was added to suppress lactation. This was done because lactation contributes to hypocalcemia via PTH-related peptide and calcium loss in milk. The T3 was then added to the medical regimen. The patient's hypothyroid state was thought to be increasing her prolactin level, again resulting in calcium loss through lactation. The T3 was chosen for its shorter half-life, knowing it would be discontinued 2 to 3 weeks before postoperative radioactive iodine treatment. Hydrochlorothiazide was added to reduce calciuria. These extensive medical maneuvers and a significantly lengthened hospital stay were required to stabilize calcium levels in this otherwise healthy young woman.

In conclusion, the incidence of thyroid cancer is increasing, and as a result, more patients are undergoing thyroid surgery for this disease. Pregnant and lactating women are included in this population, especially as thyroid masses are increasingly recognized during pregnancy. Such patients can have significantly altered calcium homeostasis. As a result, postoperative hypoparathyroidism can result in severe hypocalcemia which is exceedingly difficult to correct and may require the assistance of colleagues in endocrinology. A better understanding of these mechanisms and the pharmacologic means to correct them will aid future patients in this setting.

\section{REFERENCES}

1. American Cancer Society: Cancer Facts and Figures 2007. Atlanta, Georgia: American Cancer Society, 2007.

2. Heller HJ, Greer LG, Haynes SD, Poindexter JR, Pak CY. Pharmacokinetic and pharmacodynamic comparison of two calcium supplements in postmenopausal women. J Clin Pharmacol 2000;40:1237-1244.

3. Hanzlik RP, Fowler SC, Fisher DH. Relative bioavailability of calcium from calcium formate, calcium citrate, and calcium carbonate. J Pharmacol Exp Ther 2005;313:12171222.

4. Reeve T, Thompson NW. Complications of thyroid surgery: how to avoid them, how to manage them, and observations on their possible effect on the whole patient. World J Surg 2000;24:971-975.

5. Kovacs CS. Calcium and bone metabolism during pregnancy and lactation. J Mammary Gland Biol Neoplasia 2005;10:105-118. 